\title{
Proposal for ontology based approach to fuzzy student model design
}

\begin{abstract}
Intelligent tutoring system (ITS) is a software system designed using artificial intelligent techniques (comprising of Fuzzy Logic, Neural-Networks, Bayesian networks, Ontology, Genetic Algorithms and Software Agents) to provide an adaptive and personalized tutoring suitable to each individual student based on his/her profile or characteristics. In this paper we intend to employ the use of Fuzzy logic and Ontology techniques to model the student's learning behaviour with the aim of improving the learning path and increase the system's adaptability. The use of fuzzy logic in this context is to enable the computational analysis of the student's characteristics and learning behaviours in order to handle the uncertainty issues related to the student model design. Ontology is a vital tool for managing knowledge in a particular domain and is one of the recent techniques used to design the representation of student's cognitive state.
\end{abstract}

Keyword: ITS; Fuzzy logic; Ontology; Student model 